\title{
A low energy-model for coherent radio emission - the three-liter-pulsar
}

\author{
Harald Lesch \\ University Observatory, Scheinerstr. 1, 81679 Munich, Germany \\ Thomas Kunzl \\ University Observatory, Scheinerstr. 1, 81679 Munich, Germany \\ Max-Planck-Institut for Extraterrestrial Physics, Giessenbachstr., 85748 \\ Garching, Germany
}

Axel Jessner

Max-Planck-Institut for Radioastronomy, Auf dem Hügel 69, 53121 Bonn, Germany

\begin{abstract}
We present a new model for the coherent radio emission of pulsars that succeeds in reproducing observed luminosities at emission heights of 50-100 pulsar radii. Based on energy conservation and propagation effects we apply the concept of a free electron maser (FEM) to the creation of coherent radio emission. With the characteristic frequency $\nu \simeq \gamma^{2} \nu_{\text {pe }}\left(\nu_{\text {pe }}\right.$ denotes the plasma frequency and $\gamma$ is the Lorentz factor of the emitting particles) we find that even for the lowest frequencies at which the highest degree of coherence is required only low energy particles $(\gamma \sim 10)$ with Goldreich-Julian-density are necessary. Because of its low energy budget we call it three-liter-pulsar.
\end{abstract}

\section{Introduction}

The observations of radio pulsars set very hard constraints on any model about the origin of the coherent emission. Especially the deduction of very small emission heights of the order of 50-100 pulsar radii (e.g. Kramer et al. 1997; Kijak and Gil 1998) is a challenge for theoretical investigations. We could show that the model of coherent curvature radiation is not able to reproduce the observed radio properties, neither the luminosities, nor the observed weak radius to frequency mapping (Lesch et al. 1998). We could also show that the assumption of a significant population of secondary pairs with densities far beyond the Goldreich-Julian density is in contradiction to the observed low-frequency radio emission (Kunzl et al. 1998b).

Given these two results we investigated the role of free electron maser action in a pulsar magnetosphere (Kunzl et al. 1999)

The FEM-mechanism requires a strong localized oscillating electric field parallel to the magnetic field, causing e.m. emission at $\omega \simeq \omega_{0} \gamma^{2}$, where $\omega_{0}$ is 
the oscillation frequency and $\gamma$ corresponds to the Lorentz factor in the range of the inversion $\frac{d f(\gamma)}{d \gamma}>0$ that pumps the FEM (Melrose 1995). Usov (1987) has investigated the consequences of discontinuous discharges on the surface of the neutron star surface. He found that such discharges are able to establish the inverted energy distribution necessary to drive the action of a FEM and the excitation of strong density fluctuations as spatially varying strong electrostatic solitary structures.

Kuijpers and Volwerk (1996) have shown that particles entering such electric field structures emit frequencies of about

$$
\nu \propto \gamma^{2} / \tau_{0}
$$

where $\tau_{0}=\gamma m_{e} c / e E_{0}$ is their characteristic interaction time of the particles with the electric field $E_{0}$. The interaction time is equivalent to the inverse of the respective frequency of the electric field. For an electric field driven by density fluctuations, $\tau_{0} \simeq \omega_{\mathrm{pe}}^{-1}$, with the electron plasma frequency $\omega_{\mathrm{pe}}$. From eqn. (1), a Lorentz factor of 10 and the peak of observed emission at $400 \mathrm{MHz}$ one would estimate $\tau_{0} \simeq 0.25 \mu \mathrm{s}$. Kuijpers and Volwerk provided the important result that this mechanism is able to generate the huge brightness temperatures (up to $10^{30} \mathrm{~K}$ ) from relativistic electrons with small Lorentz factors $(\gamma$ a few), which is in full accordance with our findings.

The FEM is a relativistic plasma emission process (e.g. Melrose 1995 and references therein). It involves two essential steps: the excitation of Langmuir waves via a two-stream instability and their subsequent nonlinear conversion into electromagnetic waves.

Langmuir waves are electrostatic longitudinal waves with frequencies close to the electron plasma frequency, which reads for relativistic electrons near the polar cap as

$$
\omega_{\mathrm{pe}}=\sqrt{\frac{n e^{2}}{\gamma m_{\mathrm{e}} \varepsilon_{0} x_{\mathrm{em}}^{3}}}
$$

with $n$ being the particle density on the pulsar surface, the Goldreich-Julian density (Goldreich and Julian 1969): $n=n_{\mathrm{GJ}}=\left(2 B \Omega \varepsilon_{0} / e\right)$, with the rotation frequency $\Omega ; x_{\text {em }}$ denoting the distance to the surface in pulsar radii, the electron mass $m_{\mathrm{e}}$ and $\gamma$ being the Lorentz factor of the electrons. In relativistic plasma emission, the maser process is the effect of a two-stream-instability caused by a $\frac{d f(\gamma)}{d \gamma}>0$ feature in the particle distribution function over a range of $\Delta \gamma$. The wave is pumped by the beam's free energy content that is a result of inverted energy population relativ to the background distribution at lower energies. This source of free energy diminishes through depopulation of the beam by the violent energy losses due to coherent radiation $\mathrm{d} f / \mathrm{d} \gamma \leq 0$ holds everywhere. This situation can be established by a beam of high energy particles moving through a background plasma. However, in the case of a continious flow the growth rates $\Gamma$ for such an instability are too small to reach wave energy densities that are high enough for nonlinear waves to develop inside the light cylinder (e.g. Usov, 1987). A larger growth rate $\left(\Gamma \propto \omega_{\text {pe }}\right)$ can result if the generation of the relativistic electrons at the surface of the neutron star fluctuates in time, producing a discontinous or modulated beam, where the faster particles overtake 
the previously emitted slower particles, which have already lost part of their energy by radiation (Usov 1987).

The relation between particle energy and the emitted frequency via free electron maser action can be deduced from the characteristic frequency of the FEM, which is $\nu=\frac{\omega}{2 \pi}=\gamma^{2} \nu_{0}$ together with (2) we obtain strongly pumped Langmuir waves at

$$
\nu(\gamma)=\gamma^{2} \frac{\omega_{\mathrm{pe}}}{2 \pi}=\sqrt{\frac{\gamma^{3} \pi n e^{2}}{m_{\mathrm{e}} \epsilon_{0} x_{\mathrm{em}}^{3}}} .
$$

$\omega_{\text {pe }}$ denotes the (frame invariant) plasma frequency of the electrons as seen in the observer's frame. Eq. (3) provides a simple connection between observed frequency, particle density and Lorentz factor. Note that this result is in agreement with the relation given by Melrose and Gedalin (1999)(hereafter MG) since we will show that the FEM mechanism works so efficiently that the particles slow down almost to rest so that the Lorentz factor of the background can be set equal to unity. Together with a very sharply peaked distribution function of the beam particles' energies (MG showed that only a hydrodynamic but not a kinetic instability can be efficient enough to explain the coherent radiation) we find $\gamma_{\mathrm{s}}=\langle\gamma\rangle=: \gamma$.

The particles lose their energy via coherent emission caused by their interaction with the spatially localized oscillating field structures that are acting as a wiggler field. The energy of the emitted electromagnetic radiation comes primarily from the particles. This leads to significant energy losses, thereby transforming the high energy beam into a low energy population which will present the background plasma for the next burst of high energy particles. According to eq.(3) rapid energy loss causes the decrease of the emitted frequency.

Eq.(3) has important consequences for the energy of the radio emitting particles: In order to get radio emission between $\nu_{\min }=100 \mathrm{MHz}$ and $\nu_{\max }=30 \mathrm{GHz}$ at a height of about $x_{\mathrm{em}}$ pulsar radii, the required Lorentz factors according to eq. (3) have to be

$$
\gamma_{\min }=4.5 \cdot 10^{-3}\left(\frac{\nu_{\min }}{\mathrm{MHz}}\right)^{2 / 3} x_{\mathrm{em}}\left(\frac{n}{n_{\mathrm{GJ}}}\right)^{-1 / 3}=4.8\left[\frac{x_{\mathrm{em}}}{50}\right]\left[\frac{\nu_{\min }}{100 \mathrm{MHz}}\right]^{2 / 3}
$$

and the same calculation yields for the highest frequency $\gamma_{\max }=216\left[\frac{x_{e m}}{50}\right]$ $\left[\frac{\nu_{\max }}{30 \mathrm{GHz}}\right]^{2 / 3}$ for $n=n_{\mathrm{GJ}}$.

Thus, the FEM requires only very low Lorentz factors. Furthermore, since the pulsar radio emission typically peaks at a few hundred $\mathrm{MHz}$, the low energy particles of some $\mathrm{MeV}$ are the preferred population for an efficient FEM-action. Regimes of either higher densities or higher $\gamma$ are not feasible: densities and particle energies are completely determined by the minimum beam energy density $W_{\text {beam }}=n \gamma m_{\mathrm{e}} c^{2} / 2$ responsible for the observed luminosity, the observed emission heights and eq. (3). As this mechanism operates only with a population of low energy particles at Goldreich-Julian densities, efficient pair production is not only ruled out but also surplus to requirement in the radio emission region.

The most important test every pulsar model has to pass is energy conservation. The total observed radio power must not exceed the available energy 
(i.e. the mean kinetic energy of one particle multiplied by the number of particles emitted from the polar cap per time unit). Of course, one has to take into account that the radiation is not emitted isotropically in the observer's frame. Therefore the peak power calculated from the observed flux is considerably higher than the real energy losses. This beaming effect has two reasons: On the one hand there is the relativistic lighthouse effect and on the other hand coherence itself produces an anisotropy in the emission as we have shown in Kunzl et al. (1998a).

\section{Energetics}

In the following discussion we shall thus restrict ourselves to the explanation of the maximum flux (at very low frequencies (a few $100 \mathrm{MHz}$ )) under the assumption that we have a fully coherent process. It is common practise in the literature to call this maximum the "turnover frequency". For the sake of brevity we will call the frequency where the highest fluxes occur peak frequency $\nu_{\text {pcak }}$. In most observed cases the spectrum has a (small) positive slope for $\nu<\nu_{\text {peak }}$ and a (larger) negative slope (mostly approximately a power law) for higher frequencies (Malofeev et al., 1994).

Knowing the connection between $\gamma$ and $\nu$ (in the observer's frame) enables us to calculate the specific power that can be radiated at the peak frequency. This relationship depends crucially on the radiation mechanism.

For the FEM mechanism, the connection between frequency and Lorentz factor reads as

$$
\gamma(\nu)=\left(\frac{2 \pi}{\omega_{\mathrm{pe}}^{(0)}} \nu\right)^{2 / 3}=4.46 \cdot 10^{-3}\left(\frac{\nu}{\mathrm{MHz}}\right)^{2 / 3} x_{\mathrm{em}}\left(\frac{P}{1 / 2 \mathrm{~s}}\right)^{1 / 3} B_{8}^{-1 / 3}
$$

in the observer's frame, which can be found by inverting (eq.(3)) under the assumption (that we will always make in the following) that the density is equal to the Goldreich-Julian density in the emission region.

Using the energy conservation argument now we find that (since the density $n$ is fixed) the power radiated in an interval $[\nu, \nu+\Delta \nu]$ is proportional to the corresponding difference of the particle energies $(\Delta \gamma)$. For infinitesimal intervals we obtain some "specific power" per frequency interval that is proportional to the measured flux in one frequency band. The important implication is, that we do not need to know any details about the radiation process apart from the relation between $\nu$ and $\gamma$ and the fact that the process is very rapid (otherwise the assumption of constant density would be doubtful).

The specific energy loss per frequency unit at $\nu=\nu_{\text {peak }}$ is

$$
\begin{aligned}
P_{\text {spez }} & =\left.A_{\text {cap }} c n_{\mathrm{GJ}} x_{\mathrm{em}}^{-3} m_{\mathrm{e}} c^{2} \cdot \frac{64}{\pi} \frac{\mathrm{d} \gamma^{5}(\nu)}{\mathrm{d} \nu}\right|_{\nu=\nu_{\text {peak }}} \\
& =54.4 \frac{\mathrm{W}}{\mathrm{Hz}}\left(\frac{P}{1 / 2 \mathrm{~s}}\right)^{-1 / 3} B_{8}^{-2 / 3}\left(\frac{\nu_{\text {crit }}}{\mathrm{MHz}}\right)^{7 / 3} x_{\mathrm{em}}^{5} .
\end{aligned}
$$

Two effects ensure that the specific radiation power is at its maximum at the lowest frequencies. As mentioned above the most reasonable scenario for the 
radiation process is that the emission starts completely incoherent so that the higher frequencies show lower brightness temperatures and the coherence factor grows until it reaches its maximum value at $\nu=\nu_{\text {crit }}$. Secondly the radiation process is very efficient so that all particles are shifted from their initial Lorentz factor $\gamma_{0}$ down to a very low value of the background particles (whose value will be derived below). Thus a fixed frequency $\nu$ is only radiated by those particles whose initial energy is equal to or larger than $\gamma(\nu)$ since their energy goes down continuously from the initial to the background value and therefore can only reach $\gamma(\nu)$ if the initial value was at least equal to that number.

Taking a steep power law with a sharp low energetic cutoff at $\gamma=\gamma_{\min }:=$ $\gamma\left(\nu_{\text {peak }}\right)$ for the particle distribution function we have only very few particles having high enough initial energies to radiate at higher frequencies but all of them can emit at $\nu_{\text {peak }}$.

\subsection{The background plasma and the beam particles}

The FEM-interaction of the relativistic particles with the fluctuating electric oscillations can be seen as a form of inverse Compton scattering. In this case an elastic interaction of a (soft) photon or a plasma wave with a relativistic electron. The typical timescale for the losses is calculated by the particle energy divided by the product of the Thomson cross section $\sigma_{\mathrm{T}}$, the particle speed and the energy density of the photons (waves). For electromagnetic (electrostatic) waves this renders: $\tau_{\text {loss }}=\frac{\gamma m_{\mathrm{e}} c^{2}}{\sigma_{\mathrm{T}} c \frac{E^{2} \varepsilon_{0}}{2}}=\frac{2 m_{\mathrm{e}} c \gamma}{\sigma_{\mathrm{T}} E^{2} \varepsilon_{0}}$. For plasma waves as considered here $E$ is given by the turbulent electric field $E_{\text {turb }}=\frac{e n_{\text {beam }} \lambda}{\varepsilon_{0}} \lambda$ being a typical scale of the dimension of the turbulent fields (here we set $\lambda=c / \omega_{\text {pe }}$ (Asseo 1993)). For $N$ coherently radiating particles $\tau_{\text {loss }}$ decreases by a factor of $N$. Taking the maximum possible coherence by setting $N:=n \lambda^{3}$ we obtain the minimum loss time for a fully coherent FEM process. Comparing this with the particle interaction time with the timescale of the localized oscillations $t_{\mathrm{pe}}=\omega_{\mathrm{pe}}^{-1}$ we obtain a ratio which depends only on the particle energy

$$
\Sigma=\frac{\tau_{\text {loss }} N_{\max }^{-1}}{t_{\mathrm{pe}}} \simeq 18.6 \gamma^{-2}
$$

This shows clearly that even for very low Lorentz factors the loss times are not considerably higher than a few plasma times. That means all frequencies are almost emitted from the same place meaning that the radius to frequency mapping is almost zero.

With eq.(7) we obtain the consistent result that even for the particles with the lowest required energy $\gamma_{\min }$ (Eq.(4)) $\Sigma \ll 1$, i.e. the particles could lose their energy well within a plasma time and within the volume occupied by the localized strong electric field of the Langmuir solitons. That makes it plausible, that indeed all particles lose their energy instantenously, i.e. (with Eq.(3)) the emission is broadband and all frequencies are emitted almost at the same place. Eq. (7) is valid only for maximum coherence, only then would the particles lose nearly all their energy within one Langmuir microstructure. However, the radiation process is bound to start with a lower degree of coherence which increases with time and reaches its maximum within a few plasma periods. For the details 
we refer to Kunzl et al. (1999). Inserting $\gamma=1$ in (7) we obtain the still small loss length of few meters corresponding to an almost vanishing RFM

We could show that for a reasonable radiated flux we need incoming fast electrons with Lorentz factors between 5 and 50 . Guided by the observed structure of the radio emission, we assume that the nonstationary discharge consists of single discharges with very small extension (close to $\lambda$ ) and that superposition of these individual pulses (so called micropulses) forms the observed pulse shape. The observations show that the micropulse flux vastly exceeds the mean flux. The ratio of micropulse to mean flux we denote with $\chi$. As a typical value we choose $\chi=1500$ as indicated by the observations (Lange et al. 1998).

The connection between $x_{\mathrm{em}}$ and $\gamma_{\mathrm{min}}$ is given by Eq. (5), $S\left(\nu_{\text {peak }}\right)$ is calculated from the observed mean flux at $408 \mathrm{MHz}$, the peak frequency and the spectral index 5 . Therefore we finally find the following expressions

$$
\gamma_{\min }=0.660\left(\frac{\nu_{\text {peak }}}{400 \mathrm{MHz}}\right)^{-(\varsigma-1) / 5}\left(\frac{P}{1 / 2 s}\right)^{2 / 5} B_{8}^{-1 / 5} \chi^{1 / 5}\left(\frac{S_{400}}{\mathrm{mJy}}\right)^{1 / 5}\left(\frac{d}{\mathrm{kpc}}\right)^{2 / 5}
$$

for the Lorentz factor and for the emission height in units of the neutron star radius

$$
x_{\mathrm{em}}=2.72\left(\frac{\nu_{\text {peak }}}{400 \mathrm{MHz}}\right)^{-(3 \varsigma+7) / 15}\left(\frac{P}{1 / 2 \mathrm{~s}}\right)^{1 / 15} B_{8}^{2 / 15} \chi^{1 / 5}\left(\frac{S_{400}}{\mathrm{mJy}}\right)^{1 / 5}\left(\frac{d}{\mathrm{kpc}}\right)^{2 / 5} .
$$

We compared the calculated emission heights with the observationally deduced ones for 45 pulsars (from Kijak and Gil 1998) and found a remarkable good agreement (Kunzl et al. 1999)

\section{References}

Asseo, E. 1993, MNRAS, 264, 940

Goldreich, P., \& Julian, W.H. 1969, ApJ 157, 869

Kijak, J., \& Gil, J.A. 1998, MNRAS 299, 855

Kramer, M., et al. 1997, A\&A, 322, 846

Kuijpers, J., \& Volwerk, M. 1996, ASP-Series 105, 181

Kunzl, T., Lesch, H., \& Jessner, A. 1998a, A\&A, 339, 917

Kunzl, T., Lesch, H., Jessner, A., \& von Hoensbroech, A. 1998b, ApJ,505, L139

Kunzl, T., Lesch, H., \& Jessner, A. 1999, ApJ (submitted)

Lange, C., Kramer, M., Wielebinski, R., \& Jessner, A. 1998, A\&A, 332, 111

Lesch, H., Jessner, A., Kramer, M., \& Kunzl, T. 1998, A\&A, 332, L21

Malofeev, V.M., et al. 1994, A\&A 285, 201

Melrose, D.B. 1995, J. Astrophys. Astron., 16, 137

Melrose, D.B., \& Gedalin, M.E., 1999, ApJ, 521, 351

Usov, V.V. 1987, ApJ 320, 333 